\title{
FACTORS INFLUENCING FUNCTIONAL OUTCOME OF PHILOS PLATE FIXATION FOR PROXIMAL HUMERUS FRACTURES
}

\author{
Hazwan bin Ab. Wahid ${ }^{1}$, Khairul Nizam bin Siron ${ }^{2}$ and Ahmad Zakiran ${ }^{3}$ \\ ${ }^{1}$ Department of Orthopaedic \& Traumatology, Hospital Tengku Ampuan Afzan, Pahang, \\ Malaysia, ${ }^{2}$ Department of Orthopaedic \& Traumatology, Kulliyyah of Medicine, \\ International Islamic University of Malaysia, Kuantan, Pahang, Malaysia, ${ }^{3}$ Klinik \\ Kesihatan Bandar Kuantan, Pahang, Malaysia.
}

Presenter: Hazwan bin Ab. Wahid, hazwan.ab.wahid@gmail.com

Introduction: Displaced and unstable proximal humerus fractures are difficult to treat and they have high morbidity. The main goal is to achieve painless shoulder with full recovery of the shoulder joint motion. Impingement syndrome is one of the commonest postoperative complication. This study aim is to appreciate the functional outcomes of Philos-plate fixation for proximal humerus fractures and to establish association with high plate positioning with impingement syndrome of the shoulder after Philos-plate fixation.

Materials and method: This is a retrospective study, reviewing 26 patients who had proximal humeral fractures and underwent open reduction and Philos-plate fixation between December 2010 and January 2015 in Hospital Tengku Ampuan Afzan, Kuantan. Preoperative X-rays or CT scans were inspected, follow-up visits and X-ray images were obtained at every follow-up to evaluate the plate position and bone union. Assessments of impingement syndrome and other complications were done and also functional outcome measured with Constant shoulder scoring system during final follow-up.

Results: Almost $70 \%$ of patients achieved good (Constant score: 71-85) to excellence (Constant score: 86-100) outcome, with 77.54 mean Constant score. All cases achieved union. None of the cases developed complications like malunion, infection, AVN, and loosening of implant while 2 cases complicated with shoulder impingement post operatively. Among all the factors, age more than 60 years, and high plate position found to be a significant factor in the outcome of the surgery where both factors yielded significantly less mean Constant scores. Neer 3 parts fractures have higher mean of Constant score compare to Neer 4 parts fractures and high plate position is significantly associated with impingement syndrome postoperatively.

Conclusion: This study showed that age, fracture type and plate position were key factors affecting shoulder function after Philos locking plate fixation surgery. We recommend that plate should be position at most $10 \mathrm{~mm}$ from the greater tubercle to prevent impingement syndrome postoperatively and for better functional outcome. 